\title{
The structural and functional mechanisms of motor recovery: complementary use of diffusion tensor and functional magnetic resonance imaging in a traumatic injury of the internal capsule
}

D J Werring, C A Clark, G J Barker, D H Miller, G J M Parker, M J Brammer, E T Bullmore, V P Giampietro, A J Thompson

\begin{abstract}
Objectives-Recovery from focal motor pathway lesions may be associated with a functional reorganisation of cortical motor areas. Previous studies of the relation between structural brain damage and the functional consequences have employed MRI and CT, which provide limited structural information. The recent development of diffusion tensor imaging (DTI) now provides quantitative measures of fibre tract integrity and orientation. The objective was to use DTI and functional MRI (fMRI) to determine the mechanisms underlying the excellent recovery found after a penetrating injury to the right capsular region.
\end{abstract}

Methods-DTI and fMRI were performed on the patient described; DTI was performed on five normal controls.

Results-The injury resulted in a left hemiplegia which resolved fully over several weeks. When studied 18 months later there was no pyramidal weakness, a mild hemidystonia, and sensory disturbance. fMRI activation maps showed contralateral primary and supplementary motor cortex activation during tapping of each hand; smaller ipsilateral primary motor areas were activated by the recovered hand only. DTI disclosed preserved structural integrity and orientation in the posterior capsular limb by contrast with the disrupted structure in the anterior limb on the injured side.

Conclusions-The findings suggest that the main recovery mechanism was a preservation of the integrity and orientation of pyramidal tract fibres. The fMRI studies do not suggest substantial reorganisation of the motor cortex, although ipsilateral pathways may have contributed to the recovery. The initial deficit was probably due to reversible local factors including oedema and mass effect; permanent damage to fibre tracts in the anterior capsular limb may account for the persistent sensory deficit. This study shows for the first time the potential value of combining fMRI and DTI together to investigate mechanisms of recovery and persistent deficit in an individual patient.

(F Neurol Neurosurg Psychiatry 1998;65:863-869)
Keywords: magnetic resonance imaging; brain mapping; motor recovery

Recovery of function, although varied in extent and time course, often follows CNS damage of vascular, inflammatory, or traumatic origin. There is now substantial evidence that cerebral functional reorganisation may occur in response to injury to the adult $\mathrm{CNS},{ }^{1-5}$ but the structural and functional bases are not fully understood. Such mechanisms are important because they help to explain the variation in clinical outcomes and may allow for the rational planning of rehabilitation strategies. Explanations that have been suggested to account for neurological recovery include (1) the resolution of acute reversible factors such as inflammation, shock, and oedema, and (2) the recruitment of undamaged brain areas resulting from release of inhibition or the formation of new connections. The second suggestion has led to the concept of cerebral "plasticity". Functional imaging using neurophysiological techniques, positron emission tomography (PET), and more recently fMRI has allowed an exploration of these dynamic processes associated with neurological recovery in vivo. Stroke has been the most widely studied, and insights into possible recovery mechanisms have been obtained, including the recruitment of cortical areas in the undamaged hemisphere ${ }^{12}$ and the expansion of representation into cortical areas adjacent to the lesion site. ${ }^{25}$ In attempts to find prognostic markers, studies have examined structural parameters including volume of infarcted tissue ${ }^{6}$ and lesion location $^{6}{ }^{7}$ as judged from CT or MRI. These imaging techniques provide no information about the integrity or directionality of fibre tracts within lesions, and furthermore lack pathological specificity.

These limitations may in part explain the absence of a strong relation between either volume of tissue damage or lesion location to motor score outcome. ${ }^{67}$

The recently developed MR technique of diffusion tensor imaging (DTI) ${ }^{89}$ allows the investigation of the orientation and integrity of brain pathways in vivo by virtue of the water diffusion characteristics within them. Information about these structural properties may complement the findings from functional imaging studies by giving insights into relations between the degree of disruption of tract 
integrity or orientation and the functional consequences.

In brain regions in which the diffusion of water varies significantly with direction, the tissue exhibits the property of anisotropy. By contrast, regions where diffusion is similar in all directions have low anisotropy (and are said to be isotropic). Anisotropy may be quantified by one of several indices including fractional anisotropy. ${ }^{9}$ These indices are derived from a full description of diffusion in the region of interest (the diffusion tensor) obtained by measuring changes in the nuclear magnetic resonance (NMR) signal with diffusion sensitisation along at least six non-collinear directions. ${ }^{8}{ }^{9}$ In an axon cylinder water diffusion is faster along the axon than across it, probably due to the presence of directional subcellular structures including the axonal membrane and the neurofilamentary cytoskeleton which behave as barriers to diffusion. ${ }^{10}$ As fibre tracts are composed of collections of similarly oriented axons, they generally exhibit high anisotropy values. ${ }^{11}$ Thus fractional anisotropy, as a measure of the directional bias of diffusion within voxels, reflects the integrity of brain fibre tracts. Furthermore, diffusion within each voxel may be most fully and naturally described by three mutually perpendicular eigenvectors, whose magnitude is given by three corresponding eigenvalues. The eigenvalues are the three principal diffusion coeffecients measured along the three (intrinsic) eigenvector directions which define the local "fibre" frame of reference for that voxel. The direction of fibres is thus given by the eigenvector of the largest eigenvalue of the diffusion tensor. ${ }^{11}$

Although a relatively recent development, it has become clear that fMRI offers an unprecedented combination of non-invasiveness, safety, and resolution in time and space with which to study brain function. ${ }^{12}$ The use of fMRI in the investigation of motor system organisation in human subjects has been well established. ${ }^{13-15}$

Focal brain injuries other than stroke have not been widely studied with either fMRI or DTI, and it is not known whether mechanisms similar to those reported in ischaemic stroke (including the recruitment of pathways in the undamaged hemisphere) are involved in their recovery. The aims of this study were therefore twofold: firstly to determine whether fMRI and DTI could be used together in one patient to give useful insights into recovery mechanisms, and secondly to apply the techniques to a specific example of recovery from a traumatic focal brain lesion to assess the contribution, if any, of brain reorganisation in this setting.

\section{Case report}

A 21 year old man had a penetrating brain injury to his right orbit as a result of an assault with a snooker cue. He lost consciousness and was initially unresponsive. When admitted to hospital he had recovered consciousness and had a dense left hemiplegia associated with sensory disturbance. Brain CT disclosed a posterior defect of the right orbit with a large intracerebral bleed extending from the right frontal lobe, through the right basal ganglia, and into the right parietal cortex. He made an excellent physical recovery over the next month. When examined 18 months later, at the time of our study, motor examination disclosed minimal dystonic posturing of the fingers of the outstretched left hand and of the left foot with a left sided reflex emphasis and extensor plantar response but no detectable pyramidal weakness. There was some mild bradykinesia with fatiguability in the left hand but no tremor. His gait was slow with reasonable armswing but mild dystonic posturing of the left foot (predominantly inversion). Some alteration of light touch sensation on the left side persisted. Cognitive testing showed mild deficits in verbal memory and impaired executive skills with abulia.

\section{Methods}

Informed written consent was obtained for all experiments. The patient was studied with fMRI and DTI. In addition, five healthy controls (mean age 28.4 (SD 5.3) years) were studied with DTI. All studies were performed on a Signa Horizon Echospeed 1.5 T scanner (General Electric, Milwaukee, USA) and all images were acquired in the axial plane with a $24 \times 24 \mathrm{~cm}$ field of view. Mildly and heavily T2 weighted images $(\mathrm{TE}=42 / 84 \mathrm{~ms}, \mathrm{TR}=2000$ ms, matrix $256 \times 256$ ) were used to assess the extent of the remaining lesion site in the patient.

\section{FUNCTIONAL MRI}

In the fMRI studies 20 second epochs of unilateral simple paced finger tapping $(2.5 \mathrm{~Hz})$ alternated with rest. T2 weighted gradient echo echoplanar imaging (EPI) data depicting blood oxygenation level dependent (BOLD) contrast ${ }^{16}$ were acquired (matrix size $96 \times 96$ ) at each of $105 \mathrm{~mm}$ thick slice locations (interslice gap $0.5 \mathrm{~mm}$ ) through motor cortex. TR was $4000 \mathrm{~ms}$, giving 5 time points per epoch at each location; a total of 120 time points (24 epochs) were collected in 8 minutes. A high resolution inversion recovery gradient echo EPI dataset ( $\mathrm{TR}=6000 \mathrm{~ms}, \mathrm{TE}=40 \mathrm{~ms}, \mathrm{TI}=200 \mathrm{~ms}, 48 \times 3$ $\mathrm{mm}$ contiguous axial slices) covering the whole brain was also acquired on which to superimpose the activation maps. Task monitoring for mirror movements and accurate performance was by direct observation (DJW). Three experiments were performed with each hand to ensure acceptable reproducibility of results. One study was rejected due to inaccurate paradigm performance. The standardised power of MR signal change at the frequency of the periodic alternation between tapping and rest conditions was estimated at each voxel by iterated least squares fit of a sinusoidal regression model as described by Bullmore et al. ${ }^{17}$ Standardised power was also estimated at each voxel after each of 10 random permutations of the observed time series. Maps were constructed representing observed and randomised power estimates at each voxel for each experiment. These maps were coregistered with a template image in the standard space of Talairach and 

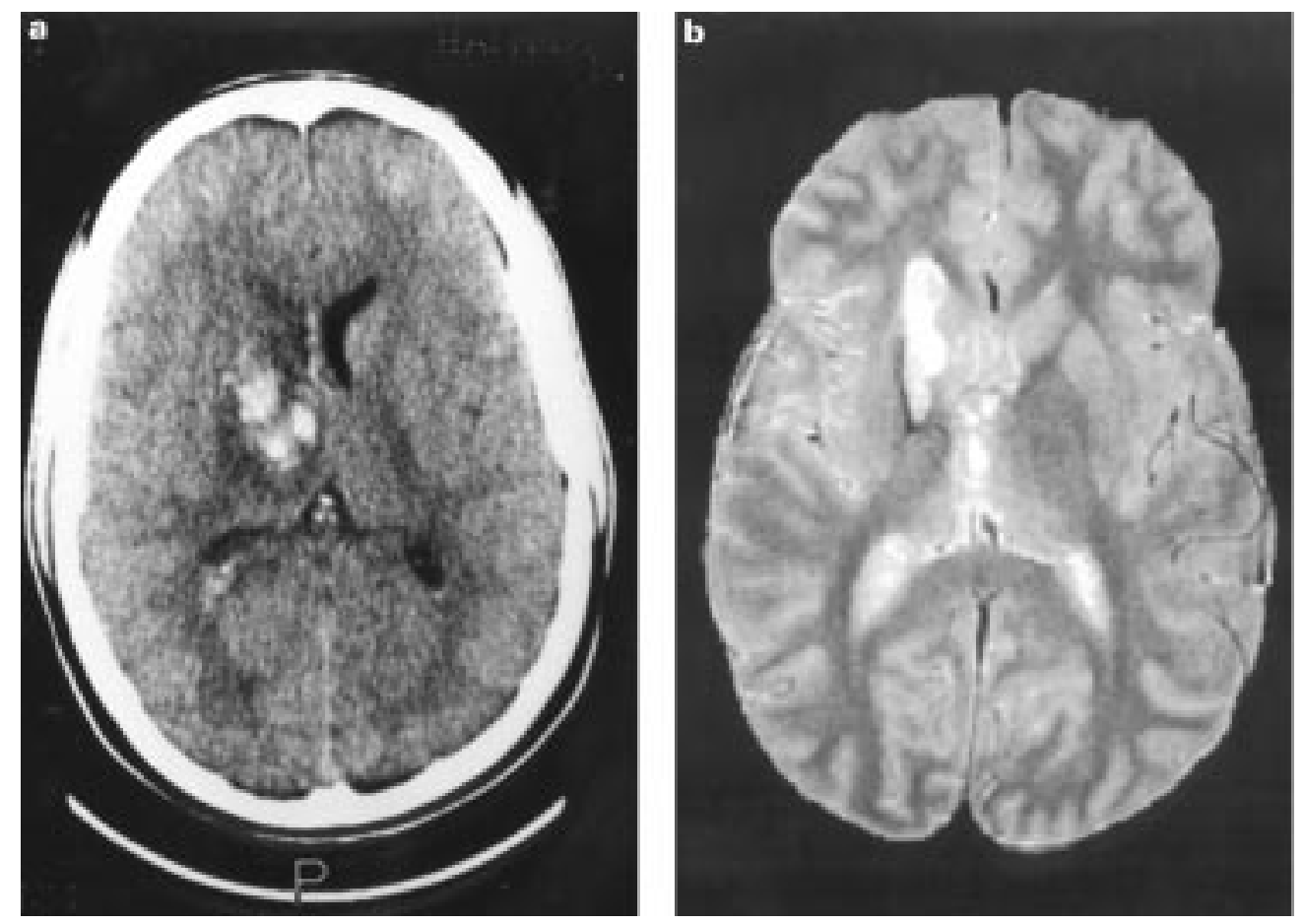

Figure 1 (a) CT taken on the day of the injury. Note high signal (haemorrhage) in the region of the right anterior internal capsule with mass effect and oedema. (b) Mildly T2 weighted MRI taken 18 months after the injury, at the time of our study. Note persistent high signal abnormality in region of right caudate and lentiform nuclei extending into genu and posterior limb of internal capsule.

Tournoux, ${ }^{18}$ and identically smoothed with a two dimensional Gaussian filter (standard deviation $=1.5 \mathrm{~mm}$ ). The median value of the standardised power was computed at each voxel of the observed maps and tested against critical values of median standardised power ascertained from the randomised maps (see Brammer et $a l^{19}$ for details of registration procedures and generic brain activation map- ping. In this study, a voxel was robustly identified as generically activated by experimental design with probability of type I error $\mathrm{p}<0.0001$. Activated voxels were colour coded (as described in the legend to figure 2) and superimposed on the grey scale backround of the patient's anatomical EPI dataset coregistered in standard space to form a generic brain activation map. ${ }^{19}$ The Talairach grid was

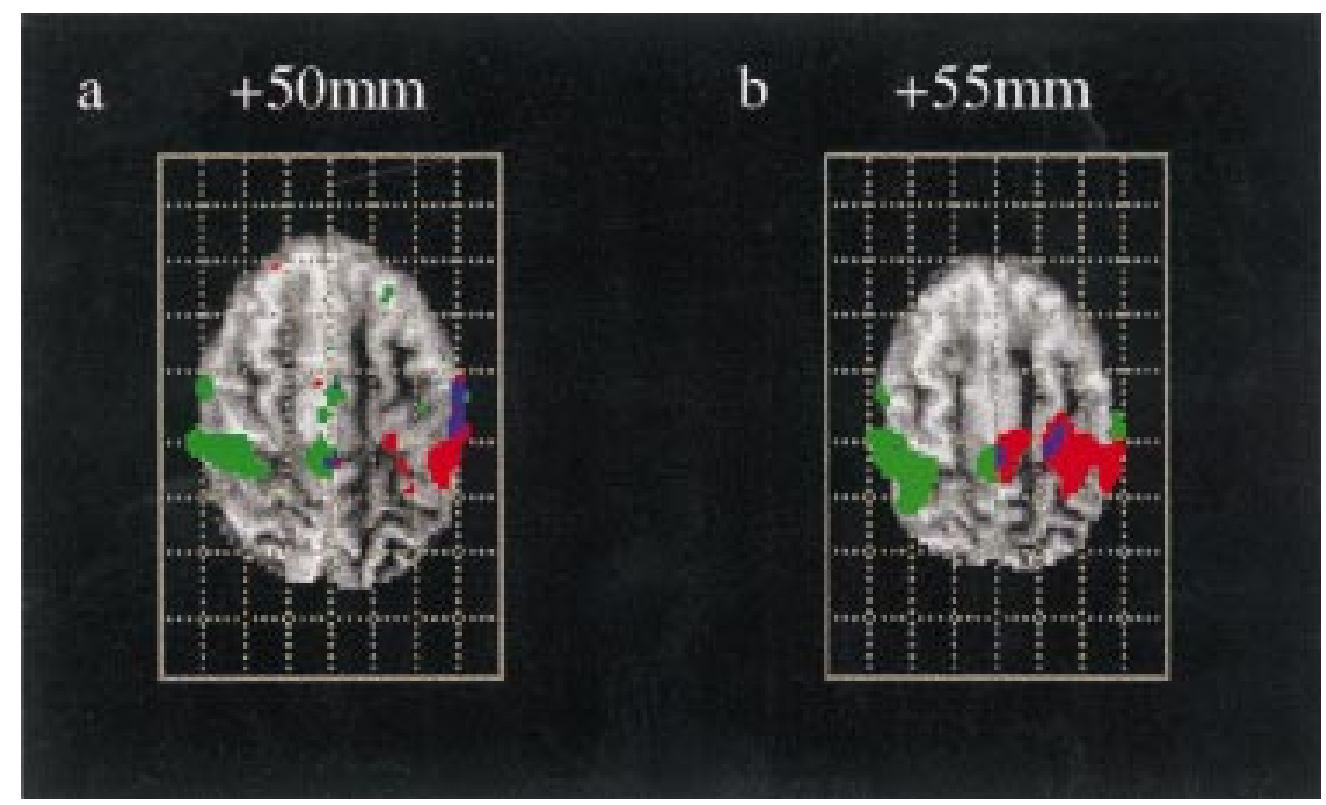

Figure 2 Generic brain activation maps derived from two axial slices of $f M R I$ data representing motor cortex $(+50$ and $55 \mathrm{~mm}$ above the intercommissural (AC-PC) line in Talairaich space). Voxels activated by right (unaffected) finger tapping are coloured red; voxels activated by left (recovered) finger tapping are shown in green; voxels activated by both right and left finger tapping are shown in blue. The voxel wise probability of type I (false positive) error $p<0.0001$. The maps are oriented with right brain represented on the left side of each panel. Note that finger tapping in both hands predominantly activates contralateral primary and supplementary motor cortices. Finger tapping in the left (recovered) hand also activates smaller areas of ipsilateral primary motor cortex. 
Table 1 Fractional anisotropy (FA) values in the patient studied and five normal controls. Note dramatic reduction in the injured anterior limb but preservation in the contralateral anterior and both posterior limbs in the patient

\begin{tabular}{|c|c|c|c|c|}
\hline \multirow[b]{2}{*}{ Subject } & \multicolumn{4}{|c|}{ Fractional anisotropy (dimensionless units) (SD) } \\
\hline & $\begin{array}{l}\text { Anterior limb } \\
\text { Right }\end{array}$ & Left & $\begin{array}{l}\text { Posterior limb } \\
\text { Right }\end{array}$ & Left \\
\hline Patient & $0.29(0.14)$ & $0.78(0.13)$ & $0.72(0.08)$ & $0.80(0.05)$ \\
\hline Control 1 & $0.76(0.07)$ & $0.82(0.10)$ & $0.82(0.08)$ & $0.81(0.08)$ \\
\hline Control 2 & $0.72(0.09)$ & $0.72(0.09)$ & $0.86(0.09)$ & $0.86(0.06)$ \\
\hline Control 3 & $0.76(0.08)$ & $0.69(0.16)$ & $0.77(0.06)$ & $0.81(0.07)$ \\
\hline Control 4 & $0.73(0.06)$ & $0.68(0.08)$ & $0.80(0.08)$ & $0.80(0.08)$ \\
\hline Control 5 & $0.74(0.06)$ & $0.81(0.07)$ & $0.82(0.10)$ & $0.85(0.08)$ \\
\hline
\end{tabular}

superimposed on the activation maps to assist anatomical localisation.

\section{DIFFUSION TENSOR IMAGING}

Diffusion weighted echoplanar images $(96 \times 96$ matrix) were acquired ( $4 \mathrm{~b}$ values increasing from $\sim 0-700 \mathrm{~s} / \mathrm{mm}^{2}$ applied in seven noncollinear directions) at 10 slice locations corresponding to a subset of those of the conventional spin echo images through the basal ganglia. These were chosen to optimally demonstrate the corticospinal tracts. These data were acquired in two blocks of five slices due to a limitation on total image storage. For each dataset the total number of images acquired was 980 . The diffusion tensor was estimated, from which fractional anisotropy values, eigenvalues, and eigenvectors were calculated in the manner described by Basser et $a l .{ }^{8}{ }^{9}$ Images were displayed on a Sun workstation (Sun microsystems, Mountain View, California, USA), and regions in the anterior and posterior internal capsule were defined on the fractional anisotropy maps with reference to the corresponding T2 weighted images. Regions were away from areas of significant geometric distortion on the echoplanar images. To display fibre directions a colour map was generated as described by Pierpaoli. ${ }^{20}$ In these maps the principle eigenvector components for each voxel $\left(\left|e_{x}\right|,\left|e_{y}\right|,\left|e_{z}\right|\right)$ are shown in red, green, or blue respectively. For greater clarity each value was modulated by fractional anisotropy, and the resulting map was overlayed on the fractional anisotropy template.

\section{Results}

The original CT at the time of injury disclosed an area of increased signal extending from the frontal lobe through the basal ganglia to parietal cortex. There was some distortion of the right lateral ventricle, oedema, and mild mass effect (fig 1a). On the MRI performed at the time of our study, 18 months later, an area of heterogeneous increased signal was seen on both heavily and mildly T2 weighted images extending from the frontal lobe into the right basal ganglia (fig 1b), involving the caudate and lentiform nuclei and the internal capsule but with some sparing of the right thalamus. fMRI activation maps (fig 2) showed activation in contralateral primary motor cortex and supplementary motor cortex in response to finger tapping of each hand. Tapping the recovered hand elicited a small area of activation in ipsilateral primary motor cortex. Accurate

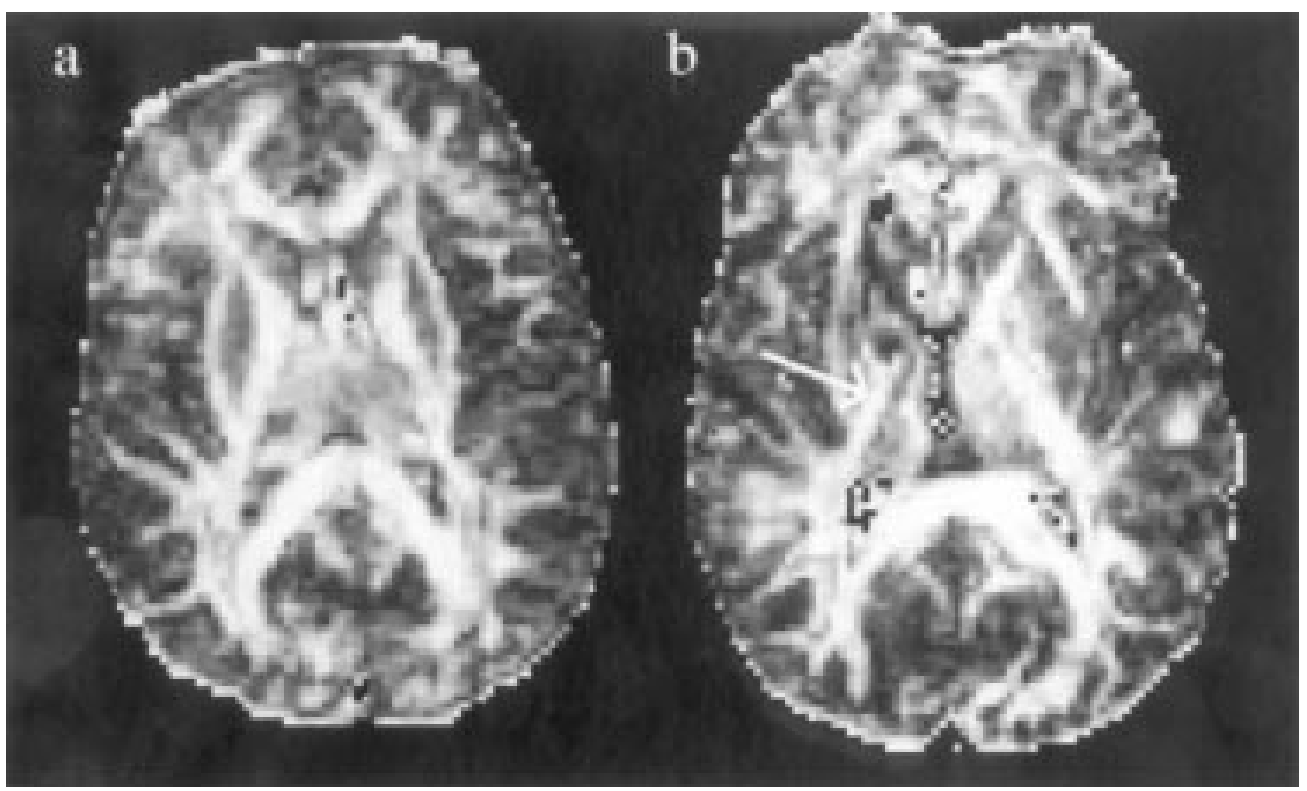

Figure 3 Fractional anisotropy maps from axial slices through the pyramidal tracts. (a) normal control and (b) patient. Note in the patient a reduction in fractional anisotropy in the anterior internal capsule on the injured side compared with the intact side, but preservation in the posterior limb of the internal capsule on the affected side in the region of the pyramidal tract. (The very bright pixels in the region of the splenium of the corpus collosum represent a loss of diffusion information. This may be accounted for by movement of the corpus callosum in the rostrocaudal axis during the cardiac cycle, which has been demonstrated by MR velocity imaging (see Feinberg and Mark ${ }^{24}$ ). We think that this motion may be due to brain pulsation. 


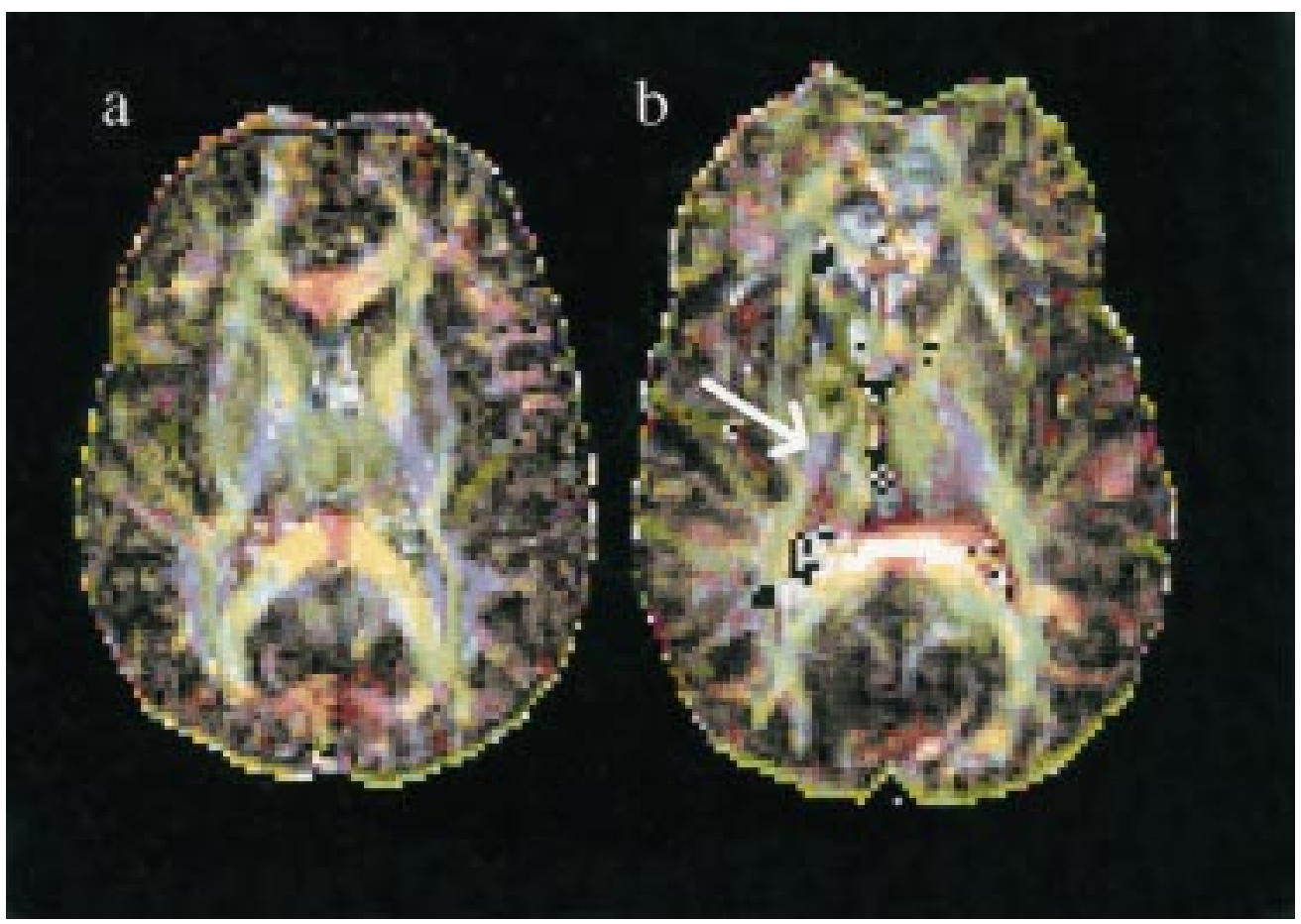

Figure 4 Colour maps in which the principle eigenvector components for each voxel $\left(\left|e_{x}\right|,\left|e_{y}\right|\right.$, and $\left.\left|e_{z}\right|\right)$, modulated by fractional anisotropy, are shown in red, green, and blue respectively. (a) normal control. Note that the predominant diffusion direction in the anterior limb of the internal capsule is in the $y$ direction (horizontally and anteroposteriorly; green). In the posterior limb it is predominantly in the $z$ direction (into the page; blue) and in the genu and splenium of the corpus callosum it is in the $x$ direction (horizontally and mediolaterally; red). (b) patient. Note dark area in the region of the injury which represents low fractional anisotropy (isotropic diffusion). In the middle third of the posterior capsular limb there is a region of preserved $z$ direction diffusion (blue) representing the intact pyramidal tract (arrowed).

mapping of basal ganglia activations was not possible due to limited brain coverage. fMRI findings from simple paced finger tapping in 10 normal subjects have been reported elsewhere. $^{21}$

Fractional anisotropy maps gave a clear demonstration of white matter tract anatomy in both controls and the patient (fig $3 a$ and $b$ ). The maps, derived from images through the level of the internal capsule and basal ganglia, showed marked loss of tissue integrity (low fractional anisotropy values) in the region of the lesion site and anterior limb of the internal capsule compared to the unaffected side. By contrast, fractional anisotropy values in the posterior limb of the internal capsule were well preserved bilaterally. All control subjects had high fractional anisotropy values in both limbs of the internal capsule (table 1). Maps derived from the diffusion tensor encoding the components of the eigenvalue of the principle eigenvector as red $\left(\left|e_{x}\right|\right)$, green $\left(\left|e_{y}\right|\right)$, and blue $\left(\left|e_{z}\right|\right)$ with modulation by fractional anisotropy were generated by the method of Pierpaoli ${ }^{20}$ (see methods section). These maps showed the dominant diffusion direction in the anisotropic posterior limb in controls to be predominantly in the through slice (z) direction (fig $4 a$ ). In the patient, a small region of preserved anisotropy with diffusion predominantly in this $(\mathrm{z})$ direction was seen in the middle third of the posterior limb of the internal capsule (fig $4 b$ ) on the affected side. The direction of anisotropy in the anterior limb of the internal capsule in controls was predominantly in the horizontal anteroposterior (y) direction. In the patient this directionality was entirely lost on the affected side but not the uninjured side.

\section{Discussion}

The patient presented is an example of neurological recovery from a focal traumatic brain injury. The dramatic early recovery seen in this case may have been due to two possible mechanisms or a combination of both: (1) resolution of reversible factors (including oedema and mass effect) affecting motor pathway function with a subsequent return of function in the intact motor tracts or (2) some form of cortical reorganisation or "plasticity" to compensate for irreversible damage to subcortical motor pathways. Our experiment aimed to determine the contributions of these mechanisms.

The fMRI maps obtained from motor tasks with either the unaffected or recovered hand suggest that similar cortical areas (mainly contralateral primary motor cortex) are involved in hand movement on each side. This in turn suggests that similar anatomical pathways (classic crossed corticospinal projections) are being utilised in each case, and that despite the nature of the right sided injury these corticospinal fibres had been spared. A region of preserved structural integrity in the middle portion of the posterior limb of the internal capsule on the patient's right side is clearly seen on the fractional anisotropy maps (fig 3b), By contrast with the reduced anisotropy (implying reduced tissue structure) in the right sided anterior capsular limb. The highest degree of diffusion in this anisotropic posterior region 
was shown to be predominantly in the $\mathrm{z}$ direction by examining the eigenvector, consistent with the expected orientation of corticospinal tract fibres. These diffusion findings therefore support the preservation of the integrity and directionality of the pyramidal tract on the injured side; this information is not obtainable from any other in vivo imaging technique. A classic view of the organisation of fibres at the level of the internal capsule is of a single homunculus in which there is a somatotopic representation of primary motor cortex areas, with the head represented in the anterior limb, the mouth in the genu, the upper limb in the anterior part, and the lower limb in the posterior part of the posterior limb. ${ }^{22}$ However, more recent studies using injections of the neuroanatomical tracer horseradish peroxidase into cortical motor areas of primates ${ }^{7}$ have challenged this model. The investigators suggest that supplementary motor area fibres descend to run horizontally in the anterior limb towards the genu. Dorsolateral premotor cortex projects via the genu, and primary motor cortex (both hand and foot areas) via the middle third of the posterior limb. Our results are in good agreement with these anatomical data: the horizontal orientation of anterior limb fibres in controls and on the uninjured side is clearly demonstrated (shown in green in fig $4 a$ and b). On the injured side the area of preserved anisotropy in the $\mathrm{z}$ direction (blue) is in the middle third of the posterior limb, in keeping with pyramidal tract preservation and the excellent motor recovery.

The same authors ${ }^{7}$ also studied 23 patients with capsular or striatocapsular strokes which they divided into those affecting four anatomical regions: (1) the basal ganglia only, (2) the anterior limb and basal ganglia, (3) the posterior limb only, and (4) the posterior limb and thalamus. They found that all groups apart from the last made an excellent motor recovery. Our patient sustained structural damage to the basal ganglia, anterior limb, and genu but less severe damage to the thalamus, so although of traumatic rather than vascular origin, might have been expected to have made a good motor recovery on this basis. Based on the clinical findings above, Fries et $a l^{7}$ challenged the traditional concept of a hierarchical motor system in which primary motor cortex projects via the corticospinal tract and is modulated by premotor and supplementary motor areas (which contribute to "planning" and "preparation" for willed movement). The authors suggest that because the supplementary motor area, premotor areas, and primary motor areas descend in parallel via the anterior, genu, and posterior capsule respectively, they may be able to substitute for each other functionally during recovery from a hemiparesis. According to this model the subject we studied would have damaged mainly the descending supplementary motor area projections, which might be expected to result in a compensatory increase in premotor and primary motor cortex activity. We did not find evidence for this mechanism (in the form of increased activation in contralateral premotor or primary motor areas on tap- ping the recovered hand) in our study. Our finding of areas of activation of the ipsilateral primary motor cortex in response to tapping the recovered hand is more suggestive of a recruitment of ipsilateral pathways as has been suggested by previous PET studies in motor stroke. ${ }^{12}$ However, the variability in lateralisation of cortical activation between the dominant and non-dominant hand may be considerable in fMRI experiments in normal subjects. ${ }^{15}{ }^{21}$ From a single case study the relevance of our fMRI findings in recovery remains undetermined.

That preservation of the pyramidal tract is a predictor of good clinical recovery has been suggested by other investigators. Binkowski et $a l^{6}$ studied motor recovery from hemiparetic ischaemic stroke in 23 patients using glucose PET and conventional MRI. They found that patients with a large volume of damage to the pyramidal tract on proton density MRI on average had a worse outcome than those in whom this tract is spared, but no correlation was found between lesion size and change in motor score. They also found, in common with Fries et $a l,{ }^{7}$ that thalamic damage (shown by hypometabolism on PET) was a predictor of poor outcome. Fractional anisotropy may prove to be a more sensitive measure of tract damage than volume of altered signal on conventional MR images, and may therefore relate more strongly to outcome after a focal lesion affecting the motor pathway. Further studies are required to consider this possibility.

The low fractional anisotropy values seen in the anterior limb of the internal capsule on the affected side reflect irreversible structural damage to fibres in this region, probably including axonal loss and a distortion of ventricular anatomy creating a CSF filled cavity. Because the tracts contained in this area are not as clinically important for limb motor control in either traditional ${ }^{22}$ or more recent ${ }^{7}$ anatomical models of capsule organisation as the posteriorly located pyramidal tracts, this structural damage has not resulted in a severe permanent disturbance of motor function. A contribution to afferent fibre tracts in the anterior limb of the internal capsule is made by thalamocortical projections which are involved in the perception of sensory information. The loss of integrity of fibres in this area is consistent with the residual sensory deficit shown in our patient. The nuclei of the basal ganglia have complex roles in both motor control and behaviour. In a review of 240 cases of lesions to the basal ganglia from various causes, Bhatia and Marsden ${ }^{23}$ found dystonia and abulia to be among the commonest symptoms, occurring in $36 \%$ and $13 \%$ of lesions respectively. Both of these symptoms were present in the patient described here, in whom both lentiform nucleus and caudate nucleus damage occurred.

A limitation of our data is the long interval between the injury and the study. This raises the possibility that during the acute recovery process some reorganisation of cortical function took place but that this had diminished by the time of our fMRI experiment. This question may only be considered by a 
longitudinal study design. DTI studies at the time of injury would have been helpful in assessing the extent and duration of the structural changes occurring at the site of trauma, and the degree and time course of recovery of pyramidal tract integrity. Although DTI has provided unique and important information about the recovery of structural integrity in the damaged corticospinal tract of the patient studied, we have not definitively proved that functional conduction in the pathway was acutely impaired at the time of injury, with subsequent resolution.

Evidence for this could be obtained using a neurophysiological technique such as transcranial magnetic stimulation, although this technique should be used with caution in focal brain injury due to the possibility of epileptogenesis. In future studies a multimodal approach incorporating non-invasive functional neuroimaging together with neurophysiology might be the most informative.

A technical limitation of our data is the resolution of the diffusion weighted images, which may introduce artifacts into the anisotropy measurements obtained. ${ }^{11}$ Our voxel dimensions $(2.5 \times 2.5 \times 5 \mathrm{~mm})$ are perhaps larger than optimal, and could result in artifacts due to partial volume (sampling of a mix of tissue types within a voxel). Furthermore, fibres with different trajectories may cross within a single voxel, causing possible misinterpretation of the diffusion tensor. However, increasing resolution results in a reduced signal to noise ratio which may also lead to errors in fractional anisotropy estimation (as fractional anisotropy is known to be noise dependent). We found that the voxel size employed in our study provided the best currently available trade off between resolution and signal to noise using our scanner.

\section{Conclusion}

We have presented the DTI and fMRI findings in a patient after a traumatic internal capsule injury. In summary, our findings suggest that the clinical recovery found in the case presented was not due to extensive cortical reorganisation, but mainly to the survival of existing motor (pyramidal) pathways. We have shown the preservation of both the integrity and direction of the pyramidal tract on the injured side using DTI, which are not visualised using standard in vivo imaging methods. Furthermore, to the best of our knowledge this is the first study to use DTI to evaluate a traumatic brain injury. The initial hemiplegia was most likely to be due to a disruption of function in the pyramidal tract due to reversible factors, probably including impaired conduction due to oedema or mass effect in the region of the injury. Irreversible damage sustained to the anterior internal capsule and basal ganglia nuclei account for the persisting contralateral sensory deficit and hemidystonia. This case study shows for the first time the potential value of combining maps of brain activation and white matter tract organisation, derived from fMRI and DTI data respectively, to elucidate the mechanisms of recovery and persistent deficit resulting from a focal brain lesion in an individual patient.

DJW is supported by the Barnwood House Trust. ETB is supported by the Wellcome trust. The NMR research unit is supported by a generous grant from the multiple sclerosis socisupported by a generous grant from the multiple sclerosis sociMacManus, Ms Kim Birnie, Ms Beth Gunn, Ms Elizabeth MacManus, Ms Kim Birnie, Ms Beth Gunn, Ms Elizabeth
Hughes, and Ms Paula Robinson for their assistance with the
MRI scanning. We also thank the normal volunteers and the patient for allowing us to perform the experiments.

1 Chollet F, Di Piero V, Wise RSJ, et al. The functional anatomy of motor recovery after stroke in humans: a study with positron emission tomography. Ann Neurol 1991;29:63-71.

2 Weiller C, Chollet F, Friston KJ, et al. Functional reorganization of the brain in recovery from capsulostriatal infarction in man. Ann Neurol 1992;33:463-72.

3 Weiller C, Ramsay SC, Wise RJS, et al. Individual patterns of functional reorganization in the human cerebral cortex of functional reorganization in the human cerebral cort

4 Danziger N, Remy P, Pidoux B, et al. A clinical and neurophysiological study of a patient with an extensive transection of the spinal cord sparing only a part of one nterolateral quadrant. Brain 1996;119:1835-48

5 Nudo RJ, Wise BM, SiFuentes F, et al. Neural substrates for the effects of rehabilitative training on motor recovery after ischemic infarct. Science 1996;272:1791-4.

6 Binkowski F, Seitz RJ, Arnold S, et al. Thalamic metabolism and corticospinal tract integrity determine motor recovery in stroke. Ann Neurol 1996;39:460-70.

7 Fries W, Danek A, Scheidtmann K, et al. Motor recovery following capsular stroke: role of descending pathways from multiple motor areas. Brain 1993;116:369-82.

8 Basser, P, Mattiello J, Le Bihan D. Estimation of the effective self-diffusion tensor from the NMR spin echo. $\mathcal{F}$ Magn Reson B 1994;103:247-54

9 Basser P, Pierpaoli, C. Microstructural and physiological features of tissues elucidated by quantitative-diffusiontensor MRI. F Magn Reson Med B 1996;111:209-19.

10 Beaulieu C, Allen PS. Determinants of anisotropic water diffusion in nerves. Magn Reson Med 1994;31:394-400

11 Pierpaoli C, Jezzard P, Basser PJ, et al. Diffusion tensor MR imaging of the human brain. Radiology 1996;201:637-48.

12 Prichard J, Cummings J. The insistent call from functional MRI. Neurology 1997;48:797-800.

13 Rao SM, Binder JR, Bandettini PA, et al. Functional magnetic resonance imaging of complex human movements. Neurology 1993;43:2311-8.

14 Rao SM, Binder JR, Hammeke TA, et al. Somatotopic mapping of the human primary motor cortex with functional magnetic resonance imaging. Neurology 1995;45:919-24.

$15 \mathrm{Kim} \mathrm{S}-\mathrm{G}$, Ashe J, Hendrich K, et al. Functional magnetic resonance imaging of motor cortex: hemispheric asymmeresonance imaging of motor cortex: hemispher

16 Kwong KK, Belliveau JW, Chesler DA, et al. Dynamic magnetic resonance imaging of human brain activity during primary sensory stimulation. Proc Natl Acad Sci USA 1992; 89:5675-9.

17 Bullmore E, Brammer M, Williams SCR, et al. Statistical methods of estimation and inference for functional MR image analysis. Magn Reson Med 1996;35: 261-77.

18 Talairach J, Tournoux. Co-planar stereotaxic atlas of the human brain. Stuttgart: Georg Thieme Verlag, 1988.

19 Brammer MJ, Bullmore ET, Simmons A, et al. Generic brain mapping in fMRI: a non-parametric approach. Magn Reson Imaging 1997;15:763-70.

20 Pierpaoli C. Oh no! One more method for colour mapping of fibre tract direction using diffusion MR imaging data. Proc. 5th ISMRM 1997: P1741.

21 Werring DJ, Barker GJ, MacManus DG, et al. Lateralisation of motor cortex activation in right-handed subjects demonstrated by functional magnetic resonance imaging [abstract]. I Neurol Neurosurg Psychiatry 1998(in press).

22 Penfield JW, Boldrey E. Somatic motor and sensory representation in the cerebral cortex of man as studied by electrical stimulation. Brain 1937;60:389-443.

23 Bhatia KP, Marsden CD. The behavioural and motor consequences of focal lesions of the basal ganglia in man. Brain 1994;117:859-76.

24 Feinberg DA, Mark AS. Human brain motion and cerebrospinal fluid circulation demonstrated with MR velocity imaging. Radiology 1987;163:793-9. 